\title{
Molecular response properties in equation of motion coupled cluster theory: A time- dependent perspective
}

Sonia Coriani’, Filip Pawłowski', Jeppe Olsen, and Poul Jørgensen

Citation: The Journal of Chemical Physics 144, 024102 (2016); doi: 10.1063/1.4939183

View online: http://dx.doi.org/10.1063/1.4939183

View Table of Contents: http://aip.scitation.org/toc/jcp/144/2

Published by the American Institute of Physics

\section{Articles you may be interested in}

Molecular response properties from a Hermitian eigenvalue equation for a time-periodic Hamiltonian

The Journal of Chemical Physics 142, 114109 (2015); 10.1063/1.4913364

Automatic active space selection for the similarity transformed equations of motion coupled cluster method The Journal of Chemical Physics 146, 074103 (2017); 10.1063/1.4976130

Perspective: Explicitly correlated electronic structure theory for complex systems

The Journal of Chemical Physics 146, 080901 (2017); 10.1063/1.4976974

Sparse maps-A systematic infrastructure for reduced-scaling electronic structure methods. II. Linear scaling domain based pair natural orbital coupled cluster theory

The Journal of Chemical Physics 144, 024109 (2016); 10.1063/1.4939030

Convergence of coupled cluster perturbation theory

The Journal of Chemical Physics 145, 224104 (2016); 10.1063/1.4971294

Stochastic multi-reference perturbation theory with application to the linearized coupled cluster method The Journal of Chemical Physics 146, 044107 (2017); 10.1063/1.4974177

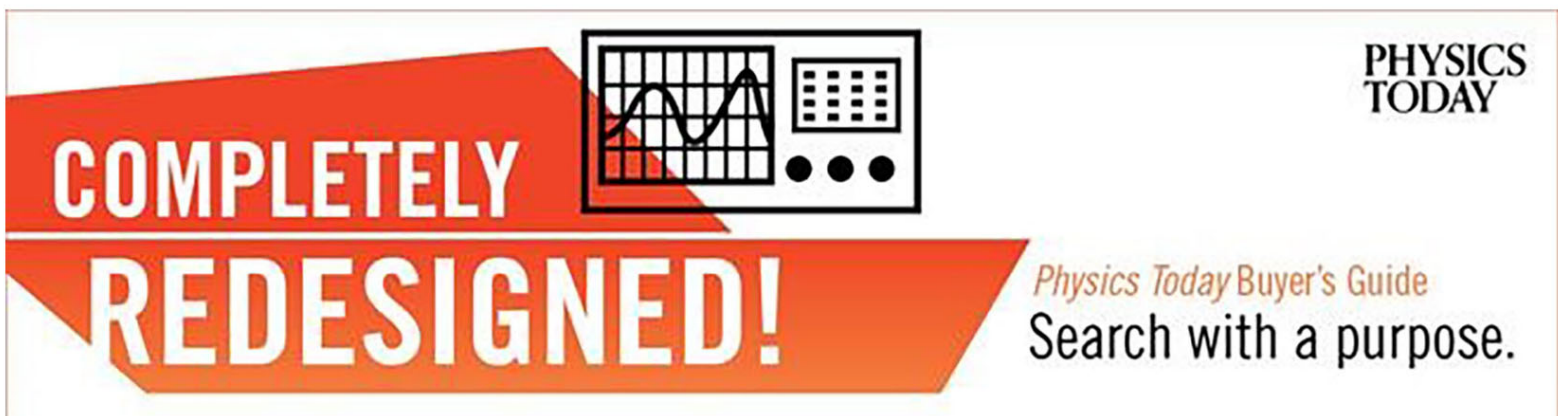




\title{
Molecular response properties in equation of motion coupled cluster theory: A time-dependent perspective
}

\author{
Sonia Coriani, ${ }^{1,2, a)}$ Filip Pawłowski, ${ }^{3,4, b)}$ Jeppe Olsen, ${ }^{3}$ and Poul Jørgensen ${ }^{3}$ \\ ${ }^{1}$ Aarhus Institute of Advanced Studies, Aarhus University, DK-8000 Aarhus C, Denmark \\ ${ }^{2}$ Dipartimento di Scienze Chimiche e Farmaceutiche, Università degli Studi di Trieste, Via L. Giorgieri 1, \\ I-34127 Trieste, Italy \\ ${ }^{3}$ qLEAP Center for Theoretical Chemistry, Department of Chemistry, Aarhus University, Langelandsgade 140, \\ DK-8000 Aarhus C, Denmark \\ ${ }^{4}$ Institute of Physics, Kazimierz Wielki University, Plac Weyssenhoffa 11, 85-072 Bydgoszcz, Poland
}

(Received 11 August 2015; accepted 17 December 2015; published online 8 January 2016)

\begin{abstract}
Molecular response properties for ground and excited states and for transitions between these states are defined by solving the time-dependent Schrödinger equation for a molecular system in a field of a time-periodic perturbation. In equation of motion coupled cluster (EOM-CC) theory, molecular response properties are commonly obtained by replacing, in configuration interaction (CI) molecular response property expressions, the energies and eigenstates of the $\mathrm{CI}$ eigenvalue equation with the energies and eigenstates of the EOM-CC eigenvalue equation. We show here that EOM-CC molecular response properties are identical to the molecular response properties that are obtained in the coupled cluster-configuration interaction (CC-CI) model, where the time-dependent Schrödinger equation is solved using an exponential (coupled cluster) parametrization to describe the unperturbed system and a linear (configuration interaction) parametrization to describe the time evolution of the unperturbed system. The equivalence between EOM-CC and CC-CI molecular response properties only holds when the $\mathrm{CI}$ molecular response property expressions-from which the EOM-CC expressions are derived - are determined using projection and not using the variational principle. In a previous article [F. Pawłowski, J. Olsen, and P. Jørgensen, J. Chem. Phys. 142, 114109 (2015)], it was stated that the equivalence between EOM-CC and CC-CI molecular response properties only held for a linear response function, whereas quadratic and higher order response functions were mistakenly said to differ in the two approaches. Proving the general equivalence between EOM-CC and CC-CI molecular response properties is a challenging task, that is undertaken in this article. Proving this equivalence not only corrects the previous incorrect statement but also first and foremost leads to a new, time-dependent, perspective for understanding the basic assumptions on which the EOM-CC molecular response property expressions are founded. Further, the equivalence between EOM-CC and CC-CI molecular response properties highlights how static molecular response properties can be obtained from finite-field EOM-CC energy calculations. ( $) 2016$ AIP Publishing LLC. [http://dx.doi.org/10.1063/1.4939183]
\end{abstract}

\section{INTRODUCTION}

The equation of motion coupled cluster (EOM-CC) eigenvalue equation was introduced by Bartlett and coworkers. ${ }^{1-3}$ This eigenvalue equation is a matrix representation of the time-independent Schrödinger equation where a coupled cluster (CC) similarity-transformed Hamiltonian, rather than the standard Hamiltonian, is used. The EOM-CC eigenvalue equation has been widely used to determine total energies for ground and excited states. ${ }^{1-13}$ The EOM-CC eigenvalue equation may be rewritten into a form where excitation energies are determined directly. ${ }^{1,3}$ In this form, the EOM-CC eigenvalue equation is identical to the Jacobian eigenvalue equation of CC response function theory ${ }^{14,15}$ for both exact $\mathrm{CC}$ states and $\mathrm{CC}$ states truncated at a given excitation

\footnotetext{
a) Electronic mail: coriani@units.it

b) Electronic mail: filip.pawlowski1@ @mail.com
}

level, as, for example, a CC singles-and-doubles (CCSD) state. The first calculation of CCSD ground-state energies was reported by Purvis and Bartlett ${ }^{16}$ and later extended to CC states where truncations were carried out at a higher excitation level. ${ }^{17-22}$ The first calculation of CCSD excitation energies was reported by Koch et $a l .{ }^{23}$ and later extended to $\mathrm{CC}$ states truncated at a higher excitation level. ${ }^{7,22,24,25}$ Excitation energies have also been determined in EOM-CC theory where CC Jacobians have been truncated using MøllerPlesset perturbation theory. ${ }^{26-32}$ Other approximate models have also been developed for determining excitation energies within the framework of EOM-CC theory and numerous applications have been reported using the EOM-CC model for calculating excitation energies. We refer to the reviews by Bartlett ${ }^{33}$ and by Krylov ${ }^{34}$ where an extensive discussion of the developments and the applications of EOM-CC theory has been presented. We also note that by appropriate choices of the basis for the EOM-CC eigenvalue equation, the EOM-CC 
eigenvalue equation can also be expressed in a form that allows the direct determination of ionization potentials and electron affinities. ${ }^{35-41}$

Molecular response properties are defined in terms of the response of an unperturbed system to a time-periodic perturbation where the response is determined by solving the time-dependent Schrödinger equation. ${ }^{42-46}$ Molecular response properties that describe the response of a ground state to a time-periodic perturbation may be expressed in terms of ground-state molecular response functions. ${ }^{47-51}$ For example, for a time-periodic electric field, first-, second-, third-, and fourth-order response properties are described by, respectively, the permanent dipole moment, the frequencydependent polarizabilities, the frequency-dependent first and second hyperpolarizabilities, and may be expressed in terms of an expectation value, linear, quadratic, and cubic response functions, respectively. Molecular property expressions that describe the strength of transitions between states, and molecular response properties of excited states may also be determined from the solution of the timedependent Schrödinger equation and explicit expressions may be determined for these properties. Examples of transition properties are the one- and two-photon transition strengths; ${ }^{52}$ an example of an excited-state molecular property is the dipole moment of an excited state. Within a response function formulation, transition strengths and excited-state molecular response properties are obtained as residues of the groundstate response functions. ${ }^{48-50}$

The EOM-CC eigenvalue equation per se does not provide information about how molecular response properties can be evaluated. However, molecular response properties have been determined in EOM-CC theory by taking CI molecular property expressions and replacing the eigenvalues and eigenstates of the CI eigenvalue equation with the corresponding eigenvalues and eigenstates of the EOM-CC eigenvalue equation. This replacement may be performed for exact wave functions as well as for wave functions that have been truncated at a given excitation level. The $\mathrm{CI} \rightarrow$ EOM-CC replacement of energies and eigenstates was first proposed by Stanton and Bartlett ${ }^{2}$ to evaluate the onephoton transition matrix elements between the ground and excited states and the dipole moments for excited states. Later, it was proposed to evaluate frequency-dependent polarizabilities ${ }^{53-55}$ and first hyperpolarizabilities ${ }^{56,57}$ using this strategy. Recently, Nanda and Krylov have applied the same strategy to determine two-photon transition matrix elements. ${ }^{58}$

The approach of Stanton and Bartlett ${ }^{2}$ for determining molecular response properties in EOM-CC theory corresponds, within a response function theory formulation, to taking CI response functions (and residues of CI response functions) in the diagonal representation and replacing the $\mathrm{CI}$ energies and eigenstates with the corresponding EOM-CC energies and eigenstates. We denote the response functions that are obtained by this $\mathrm{CI} \rightarrow \mathrm{EOM}-\mathrm{CC}$ replacement of eigenvalues and eigenstates as "EOM-CC response functions." A large variety of molecular response properties have been successfully determined from EOM-CC response functions and the residues of these response functions. ${ }^{7,15,54-66}$
In the approach by Stanton and Bartlett, ${ }^{2}$ response functions that are valid in CI theory are thus used to obtain response functions that can be used in CC theory. CI and $\mathrm{CC}$ theory are, however, very different. In the context of determining response functions, we note the following.

1. CI response functions are derived under the presumption that a linear parametrization is used to describe both the unperturbed state and its time evolution.

Contrary:

In EOM-CC theory, the unperturbed state is described using an exponential parametrization due to the replacement of $\mathrm{CI}$ eigenstates with EOM-CC eigenstates, whereas the time evolution of the unperturbed state is described by a linear parametrization since the structural form of the $\mathrm{CI}$ response functions is retained in the EOM-CC response functions.

2. In CI theory, the variational condition is satisfied and used to determine CI amplitudes.

Contrary:

In CC theory, projection is used to determine the cluster amplitude equations.

3. In CI theory, the left eigenstates of the CI eigenvalue equation are the adjoints of the right eigenstates.

Contrary:

For the EOM-CC eigenvalue equation, the left eigenstates are not adjoints of the right eigenstates.

A question therefore arises: What implications do the above differences between CI and CC theory have for the molecular response functions that are obtained in EOM-CC theory? For example, does one obtain FCI molecular response properties when no truncations are performed in the $\mathrm{CC}$ state that enters the EOM-CC eigenvalue equation? Furthermore, are molecular response properties size extensive, ${ }^{15,54}$ when they are obtained using truncated excitation manifolds?

As discussed above, CI response functions are obtained from the solution to the time-dependent Schrödinger equation using a linear parametrization to describe both the unperturbed system and its time-evolution, whereas in EOM-CC theory, the unperturbed system is described in terms of an exponential parametrization, and its time evolution is described using a linear parametrization. In Ref. 51, we therefore introduced the coupled cluster-configuration interaction (CC-CI) model where the unperturbed system is described using an exponential parametrization and the time evolution of the unperturbed state is described using a linear parametrization. We further derived explicit expressions for the CC-CI linear, quadratic, and cubic response functions using the response function theory framework developed also in Ref. 51. For a static perturbation, we also showed in Ref. 51 that finite-field EOM-CC energy calculations give CC-CI molecular response properties.

Response functions for a CI wave function may be derived using either a variational or a projection form of the time-dependent Schrödinger equation. ${ }^{51}$ When the projection method is used, an operator that symmetrizes the response functions with respect to a simultaneous frequency sign inversion and complex conjugation is introduced, thereby yielding CI response functions that are identical to those obtained using the variational condition. ${ }^{51}$ In Ref. 51, 
we showed that when the $\mathrm{CI} \rightarrow$ EOM-CC replacement of eigenstates and eigenvalues is performed for a CI linear response function that is derived by projection, the obtained EOM-CC linear response function becomes identical to the CC-CI linear response function. To see this, equivalence required a substantial rewrite of the involved response functions. In Ref. 51, we also stated that the equivalence between the EOM-CC response functions and the $\mathrm{CC}$ CI response functions was only valid for linear response functions, but did not hold for quadratic and higher-order response functions. This latter statement is incorrect and in this article, we show explicitly that molecular response properties obtained from $\mathrm{CC}$-CI response functions and EOM-CC response functions are identical, provided that the latter are obtained from CI response functions derived using projection. EOM-CC molecular response properties may therefore be obtained from response functions and residues of response functions that are determined within the CC-CI framework. ${ }^{51}$ This offers a first principle way of deriving analytic expressions for EOM-CC molecular response properties, and a time-dependent perspective to examine and understand the basic assumptions that are used to obtain EOM-CC molecular response properties.

In standard $\mathrm{CC}$ response function theory, an exponential parametrization is used to describe both the unperturbed system and its time evolution. ${ }^{14,49,51}$ As a consequence, molecular response properties that are obtained using standard $\mathrm{CC}$ response function theory are size extensive. In CC-CI theory, the unperturbed state is described by an exponential parametrization and the time evolution of this state is described by a linear parametrization and therefore the obtained molecular response properties will not be size extensive. ${ }^{51}$

The article is organized as follows. In Sec. II, the general background of EOM-CC theory is summarized. In Sec. III, static molecular response properties are introduced in terms of finite field EOM-CC energy calculations. In Sec. IV, CC-CI response functions are derived both in the elementary basis of the EOM-CC eigenvalue equation (Sec. IV A) and in the diagonal basis of the EOM-CC eigenvalue equation (Sec. IV B). In Sec. IV C, CI response functions are derived using projection in the diagonal basis of the $\mathrm{CI}$ eigenvalue equation and it is further shown that CC-CI response functions in the diagonal basis of the EOM-CC eigenvalue equation are obtained when in CI response functions, the eigenvalues and eigenstates of the $\mathrm{CI}$ eigenvalue equation are replaced by the eigenvalues and eigenstates of the EOM-CC eigenvalue equation. In Sec. $\mathrm{V}$, numerical examples are presented to illustrate that EOM-CC and CC-CI molecular response properties are identical, and in Sec. VI, we summarize our development and give some concluding remarks.

\section{THE EOM-CC EIGENVALUE EQUATION}

\section{A. The EOM-CC eigenvalue equation in the elementary basis}

Consider a CC parametrization of the ground state,

$$
\left|0_{0}^{\mathrm{CC}}\right\rangle=e^{T_{0}}|\mathrm{HF}\rangle,
$$

where $|\mathrm{HF}\rangle$ is the Hartree-Fock reference state, $T_{0}$ $=\sum_{\mu_{k}} t_{\mu_{k}} \tau_{\mu_{k}}$ is the cluster operator, $t_{\mu_{k}}$ are the cluster amplitudes, and $\tau_{\mu_{k}}$ are the many-body excitation operators that carry out excitations from $|\mathrm{HF}\rangle$ to its orthogonal complement set of states, $\left|\mu_{k}\right\rangle=\tau_{\mu_{k}}|\mathrm{HF}\rangle$, where $k$ denotes an excitation level and $\mu_{k}$ an excitation at this level. The Hartree-Fock state together with its orthogonal complement set of states form a complete set,

$$
|\mathcal{B}\rangle=\left\{|\mathrm{HF}\rangle,\left|\mu_{k}\right\rangle\right\} .
$$

The cluster amplitudes satisfy the $\mathrm{CC}$ amplitude equations and determine the $\mathrm{CC}$ ground state energy,

$$
\begin{gathered}
\left\langle\mu_{k}\left|H_{0}^{T_{0}}\right| \mathrm{HF}\right\rangle=0, \\
\left\langle\mathrm{HF}\left|H_{0}^{T_{0}}\right| \mathrm{HF}\right\rangle={ }^{\mathrm{CC}} E_{0},
\end{gathered}
$$

where we have introduced the $\mathrm{CC}$ similarity-transformed Hamiltonian, ${ }^{3,67}$

$$
H_{0}^{T_{0}}=e^{-T_{0}} H_{0} e^{T_{0}} .
$$

Introducing the biorthonormal basis (referred to as elementary)

$$
\left|\mathcal{B}^{T_{0}}\right\rangle=\left\{e^{T_{0}}|\mathrm{HF}\rangle, e^{T_{0}}\left|\mu_{k}\right\rangle\right\}, \quad\left\langle\mathcal{B}^{T_{0}}\right|=\left\{\langle\mathrm{HF}| e^{-T_{0}},\left\langle\mu_{k}\right| e^{-T_{0}}\right\},
$$

the EOM-CC eigenvalue equation becomes a matrix representation of the time-independent Schrödinger equation,

$$
\begin{aligned}
& \mathbf{H}_{0} \mathbf{C}=\mathbf{C} \mathbf{E}, \\
& \overline{\mathbf{C}} \mathbf{H}_{0}=\mathbf{E} \overline{\mathbf{C}} .
\end{aligned}
$$

The Hamiltonian matrix, $\mathbf{H}_{0}$, is non-symmetric and has the block structure,

$$
\mathbf{H}_{0}=\left(\begin{array}{cc}
\left\langle\mathrm{HF}\left|H_{0}^{T_{0}}\right| \mathrm{HF}\right\rangle & \left\langle\mathrm{HF}\left|H_{0}^{T_{0}}\right| v_{m}\right\rangle \\
\left\langle\mu_{k}\left|H_{0}^{T_{0}}\right| \mathrm{HF}\right\rangle & \left\langle\mu_{k}\left|H_{0}^{T_{0}}\right| v_{m}\right\rangle
\end{array}\right),
$$

where the $\left\langle\mu_{k}\left|H_{0}^{T_{0}}\right| \mathrm{HF}\right\rangle$ block vanishes as Eq. (3) is satisfied. $\mathbf{E}$ is a diagonal matrix containing the ground- $\left({ }^{{ }^{C}} E_{0}\right)$ and excited-state $\left({ }^{\mathrm{CC}} E_{1},{ }^{C} E_{2}, \ldots\right)$ energies on the diagonal. The left and right eigenvectors are assumed to satisfy the biorthonormalization condition,

$$
\overline{\mathbf{C}} \mathbf{C}=\mathbf{I} \text {. }
$$

We will refer to Eqs. (7)-(9) as the EOM-CC eigenvalue equation in the elementary basis ${ }^{1-3}$ of Eq. (6).

\section{$B$. The EOM-CC eigenvalue equation in the diagonal basis}

The right ground state, $\left|0_{0}^{\mathrm{CC}}\right\rangle$ [Eq. (1)], is in accordance with Eqs. (3) and (4) represented in the basis $\left|\mathcal{B}^{T_{0}}\right\rangle$ as

$$
\mathbf{C}_{0}=\left(\begin{array}{l}
1 \\
\mathbf{0}
\end{array}\right)
$$

while the left ground state, $\left\langle 0_{0}^{\mathrm{CC}}\right|$, is unit-normalized against the right state and therefore is represented in the basis $\left\langle\mathcal{B}^{T_{0}}\right.$ as

$$
\overline{\mathbf{C}}_{0}=\left(\begin{array}{ll}
1 & \overline{\mathbf{C}}_{0}^{\perp}
\end{array}\right),
$$


where $\overline{\mathbf{C}}_{0}^{\perp}$ represents the orthogonal complement component of the left ground state with elements $\bar{C}_{0 \mu_{k}}^{\perp}$ satisfying the standard CC multipliers equation,

$$
\sum_{\mu_{k}} \bar{C}_{0 \mu_{k}}^{\perp}{ }^{\mathrm{CC}} J_{\mu_{k} v_{m}}=-\left\langle\mathrm{HF}\left|H_{0}^{T_{0}}\right| v_{m}\right\rangle
$$

where we have introduced the CC Jacobian,

$$
{ }^{\mathrm{CC}} J_{\mu_{k} v_{m}}=\left\langle\mu_{k}\left|\left[H_{0}^{T_{0}}, \tau_{v_{m}}\right]\right| \mathrm{HF}\right\rangle .
$$

The left ground state may thus be written as

$$
\left\langle 0_{0}^{\mathrm{CC}}\right|=\langle\mathrm{HF}| e^{-T_{0}}+\sum_{\mu_{k}} \bar{C}_{0 \mu_{k}}^{\perp}\left\langle\mu_{k}\right| e^{-T_{0}} .
$$

The right and left excited state eigenvectors of the timeindependent Schrödinger equation are in the basis $\left|\mathcal{B}^{T_{0}}\right\rangle$ and $\left\langle\mathcal{B}^{T_{0}}\right|$ given by

$\left|0_{n}^{\mathrm{CC}}\right\rangle=-\sum_{\mu_{k}} \bar{C}_{0 \mu_{k}}^{\perp} C_{\mu_{k} n}^{\perp} e^{T_{0}}|\mathrm{HF}\rangle+\sum_{\mu_{k}} C_{\mu_{k} n}^{\perp} e^{T_{0}}\left|\mu_{k}\right\rangle, n>0$,

$$
\left\langle 0_{n}^{\mathrm{CC}}\right|=\sum_{\mu_{k}} \bar{C}_{n \mu_{k}}^{\perp}\left\langle\mu_{k}\right| e^{-T_{0}}, \quad n>0,
$$

and together with $\left|0_{0}^{\mathrm{CC}}\right\rangle$ [Eq. (1)] and $\left\langle 0_{0}^{\mathrm{CC}}\right|$ [Eq. (14)] form a biorthonormal basis,

$$
\begin{aligned}
& \left|\mathcal{B}^{\mathrm{d}}\right\rangle=\left\{\left|0_{0}^{\mathrm{CC}}\right\rangle,\left|0_{n}^{\mathrm{CC}}\right\rangle, \quad n>0\right\}, \\
& \left\langle\mathcal{B}^{\mathrm{d}}\right|=\left\{\left\langle 0_{0}^{\mathrm{CC}}\right|,\left\langle 0_{n}^{\mathrm{CC}}\right|, \quad n>0\right\},
\end{aligned}
$$

in which the EOM-CC eigenvalue equation is expressed in a diagonal form,

$$
\begin{gathered}
\left\langle 0_{p}^{\mathrm{CC}}\left|H_{0}\right| 0_{q}^{\mathrm{CC}}\right\rangle={ }^{{ }^{C} C} E_{q} \delta_{p q}, \quad p, q=0,1,2, \ldots, \\
\left\langle 0_{p}^{\mathrm{CC}} \mid 0_{q}^{\mathrm{CC}}\right\rangle=\delta_{p q}, \quad p, q=0,1,2, \ldots
\end{gathered}
$$

The elementary basis $\left[\left|\mathcal{B}^{T_{0}}\right\rangle\right.$ of Eq. (6)] and the diagonal basis $\left[\left|\mathcal{B}^{\mathrm{d}}\right\rangle\right.$ of Eq. (17a)] are connected via a non-singular transformation and therefore span the same space. This is also true for excitation manifolds of the $\mathrm{CC}$ state that are truncated at a given excitation level.

\section{EOM-CC STATIC MOLECULAR PROPERTIES BY FINITE FIELD CALCULATIONS}

In this section, we discuss how static molecular response properties consistent with the EOM-CC eigenvalue equation [Eq. (7)] may be obtained as derivatives of $E_{0}(\epsilon)$ with respect to the field strength, $\epsilon$, where $E_{0}(\epsilon)$ is a ground-state solution to the EOM-CC eigenvalue equation containing a field-dependent Hamiltonian,

$$
H(\epsilon)=H_{0}+\epsilon X,
$$

where $X$ is a Hermitian time-independent one-electron operator. The EOM-CC eigenvalue equation containing $H(\epsilon)$ becomes

$$
\left(\mathbf{H}_{0}+\epsilon \mathbf{X}\right) \mathbf{C}_{0}(\epsilon)=\mathbf{C}_{0}(\epsilon) E_{0}(\epsilon)
$$

where $\mathbf{H}_{0}$ is given in Eq. (8) and the matrix representation of the perturbation operator is given by

$$
\mathbf{X}=\left(\begin{array}{cc}
\left\langle\mathrm{HF}\left|X^{T_{0}}\right| \mathrm{HF}\right\rangle & \left\langle\mathrm{HF}\left|X^{T_{0}}\right| v_{m}\right\rangle \\
\left\langle\mu_{k}\left|X^{T_{0}}\right| \mathrm{HF}\right\rangle & \left\langle\mu_{k}\left|X^{T_{0}}\right| v_{m}\right\rangle
\end{array}\right) .
$$

Numerical differentiation of $E_{0}(\epsilon)$ gives the EOM-CC static molecular response properties. In Ref. 51, we have shown how the analytic analogue of the EOM-CC finite field calculations may be obtained using the CC-CI model, and analytic expressions have been derived for first-, second-, third-, and fourth-order molecular response properties.

\section{MOLECULAR RESPONSE PROPERTIES}

In this section, we describe how EOM-CC molecular response properties may be obtained using a time-dependent framework. We consider a molecular system described by a time-periodic Hamiltonian,

$$
H(t, \boldsymbol{\epsilon})=H_{0}+V(t, \boldsymbol{\epsilon}),
$$

where a Hermitian time-periodic perturbation with the period $T$,

$$
\begin{gathered}
V(t, \boldsymbol{\epsilon})=\sum_{j} X_{j} \epsilon_{X_{j}}\left(\omega_{X_{j}}\right) \exp \left(-i \omega_{X_{j}} t\right), \\
V(t+T, \boldsymbol{\epsilon})=V(t, \boldsymbol{\epsilon})
\end{gathered}
$$

is added to the unperturbed system. In Eq. (23), $X_{j}$ is a Hermitian time-independent one-electron operator and $\epsilon_{X_{j}}\left(\omega_{X_{j}}\right)$ is the associated perturbation strength for the real frequency $\omega_{X_{j}}$. $\boldsymbol{\epsilon}$ denotes a set of perturbation strengths $\epsilon_{X_{j}}\left(\omega_{X_{j}}\right)$. In Ref. 51, we have shown that the time-dependent Schrödinger equation for the time-periodic Hamiltonian, $H(t, \boldsymbol{\epsilon})$, may be recasted into an eigenvalue equation,

$$
\begin{aligned}
& \left(H(t, \boldsymbol{\epsilon})-i \frac{\partial}{\partial t}-\dot{\mathcal{F}}_{P_{0}}(t, \boldsymbol{\epsilon})-i \frac{\mathrm{d}}{\mathrm{d} t} \ln N(t, \boldsymbol{\epsilon})\right)\left|0_{I}(t, \boldsymbol{\epsilon})\right\rangle \\
& =\mathcal{E}(\boldsymbol{\epsilon})\left|0_{I}(t, \boldsymbol{\epsilon})\right\rangle,
\end{aligned}
$$

where $\mathcal{E}(\boldsymbol{\epsilon})$ is the quasi-energy, $\mathcal{F}_{P_{0}}(t, \boldsymbol{\epsilon})$ is the time-periodic part of the phase of the wave function, $N(t, \boldsymbol{\epsilon})$ is a real timeperiodic normalization constant, and the eigenstate $\left|0_{I}(t, \boldsymbol{\epsilon})\right\rangle$ is intermediate normalized against a reference and is timeperiodic.

\section{A. $\mathrm{CC}-\mathrm{Cl}$ molecular response properties in elementary basis}

In the CC-CI model, the unperturbed system is described by a CC state and the time evolution of this state is described using a linear parametrization. The perturbation-induced time evolution of $\left|0_{I}(t, \boldsymbol{\epsilon})\right\rangle$ may therefore be expanded linearly in the elementary basis [Eq. (6)] leading to the CC-CI wave function, ${ }^{51}$

$$
\left|0_{I}^{\mathrm{CC}-\mathrm{CI}}(t, \boldsymbol{\epsilon})\right\rangle=e^{T_{0}} e^{S(t, \boldsymbol{\epsilon})}|\mathrm{HF}\rangle,
$$


where the operator $S(t, \boldsymbol{\epsilon})$ is expanded in the state-transfer operators,

$$
S(t, \boldsymbol{\epsilon})=\sum_{\mu_{k}} s_{\mu_{k}}(t, \boldsymbol{\epsilon})\left|\mu_{k}\right\rangle\langle\mathrm{HF}|,
$$

where $s_{\mu_{k}}(t, \boldsymbol{\epsilon})$ are time-periodic expansion coefficients. Substituting $S(t, \boldsymbol{\epsilon})$ of Eq. (26) in Eq. (25) and using that $S^{2}(t, \boldsymbol{\epsilon})=0$ gives

$$
\left|0_{I}^{\mathrm{CC}-\mathrm{CI}}(t, \boldsymbol{\epsilon})\right\rangle=e^{T_{0}}|\mathrm{HF}\rangle+\sum_{\mu_{k}} s_{\mu_{k}}(t, \boldsymbol{\epsilon}) e^{T_{0}}\left|\mu_{k}\right\rangle,
$$

showing that the CC-CI wave function of Eq. (25) indeed describes a linear expansion of the time evolution in the elementary basis [Eq. (6)]. $\left|0_{I}^{\mathrm{CC}-\mathrm{CI}}(t, \boldsymbol{\epsilon})\right\rangle$ is intermediate normalized against the $|\mathrm{HF}\rangle$ reference state,

$$
\left\langle\mathrm{HF} \mid 0_{I}^{\mathrm{CC}-\mathrm{CI}}(t, \boldsymbol{\epsilon})\right\rangle=1 .
$$

Substituting Eq. (25) in Eq. (24), multiplying from the left by $e^{-S(t, \epsilon)} e^{-T_{0}}$ and projecting against $\langle\mathcal{B}|=(|\mathcal{B}\rangle)^{\dagger}$ [Eq. (2)] in the composite Hilbert space, ${ }^{51,68}$ gives the quasi-energy and the amplitude equation for determining the time-periodic expansion coefficients $s_{\mu_{k}}(t, \boldsymbol{\epsilon})$ (see Ref. 51 for a detailed derivation),

$$
\begin{aligned}
& \mathcal{E}(\boldsymbol{\epsilon})=\operatorname{Re}\left\{\left\langle\mathrm{HF}\left|e^{-S(t, \boldsymbol{\epsilon})}\left(H^{T_{0}}(t, \boldsymbol{\epsilon})-i \frac{\partial}{\partial t}\right) e^{S(t, \boldsymbol{\epsilon})}\right| \mathrm{HF}\right\rangle\right\}_{T}, \\
& \left\{\left\langle\mu_{k}\left|e^{-S(t, \boldsymbol{\epsilon})}\left(H^{T_{0}}(t, \boldsymbol{\epsilon})-i \frac{\partial}{\partial t}\right) e^{S(t, \boldsymbol{\epsilon})}\right| \mathrm{HF}\right\rangle\right\}_{T}=0,
\end{aligned}
$$

where $\{.\}_{T}$ denotes time averaging over one period $T$.

To determine molecular response properties from the quasi-energy, $\mathcal{E}(\boldsymbol{\epsilon})$, we have introduced in Ref. 51 a complex quasi-energy Lagrangian, where the amplitude equations are added to the quasi-energy in terms of the undetermined Lagrangian multipliers, $\bar{s}_{\mu_{k}}(t, \boldsymbol{\epsilon})$. When the time-evolution of the intermediate-normalized state is expressed in the elementary basis, the Lagrangian reads [cf. Eq. (29)],

$$
{ }^{c} \mathcal{L}=\left\{\left\langle\mathrm{HF}\left|e^{-S(t, \boldsymbol{\epsilon})}\left(H^{T_{0}}(t, \boldsymbol{\epsilon})-i \frac{\partial}{\partial t}\right) e^{S(t, \boldsymbol{\epsilon})}\right| \mathrm{HF}\right\rangle\right\}_{T}+\sum_{\mu_{k}}\left\{\bar{s}_{\mu_{k}}(t, \boldsymbol{\epsilon})\left\langle\mu_{k}\left|e^{-S(t, \boldsymbol{\epsilon})}\left(H^{T_{0}}(t, \boldsymbol{\epsilon})-i \frac{\partial}{\partial t}\right) e^{S(t, \boldsymbol{\epsilon})}\right| \mathrm{HF}\right\rangle\right\}_{T},
$$

where the Lagrangian satisfies the stationary conditions that determine the amplitude and multiplier equations,

$$
\begin{aligned}
& \frac{\partial^{c} \mathcal{L}}{\partial \bar{s}_{\mu_{k}}\left(\omega_{K}\right)}=0, \\
& \frac{\partial^{c} \mathcal{L}}{\partial s_{\mu_{k}}\left(\omega_{K}\right)}=0,
\end{aligned}
$$

where $s_{\mu_{k}}\left(\omega_{K}\right)$ and $\bar{s}_{\mu_{k}}\left(\omega_{K}\right)$ are amplitudes and multipliers in the frequency domain, respectively, and $\omega_{K}$ is any combination of the frequencies $\omega_{X_{j}}$ in Eq. (23). ${ }^{51}$ The perturbation components of the quasi-energy Lagrangian are defined as derivatives of ${ }^{c} \mathcal{L}$ with respect to perturbation strengths, ${ }^{51}$

$$
\begin{aligned}
& { }^{c} \mathcal{L}^{X_{j_{1}} \cdots X_{j_{n}}}\left(\omega_{X_{j_{1}}}, \ldots, \omega_{X_{j_{n}}}\right) \\
& =\left.\frac{\mathrm{d}^{n c} \mathcal{L}}{\mathrm{d} \epsilon_{X_{j_{1}}}\left(\omega_{X_{j_{1}}}\right) \cdots \mathrm{d} \epsilon_{X_{j_{n}}}\left(\omega_{X_{j_{n}}}\right)}\right|_{\epsilon=\mathbf{0}},
\end{aligned}
$$

and molecular response properties are obtained as ${ }^{51}$

$$
\begin{aligned}
& \left\langle\left\langle X_{j_{1}} ; X_{j_{2}}, \ldots, X_{j_{n}}\right\rangle\right\rangle_{\omega_{X_{j_{2}}}, \ldots, \omega_{X_{j_{n}}}} \\
& \quad=\frac{1}{2} C^{ \pm \omega c} \mathcal{L}^{X_{j_{1}} \cdots X_{j_{n}}}\left(\omega_{X_{j_{1}}}, \ldots, \omega_{X_{j_{n}}}\right), \quad \sum_{m=1}^{n} \omega_{X_{j_{m}}}=0,
\end{aligned}
$$

where $^{51}$

$$
\begin{aligned}
C^{ \pm \omega c} \mathcal{L}^{X_{j_{1}} \cdots X_{j_{n}}}\left(\omega_{X_{j_{1}}}, \ldots, \omega_{X_{j_{n}}}\right) \\
={ }^{c} \mathcal{L}^{X_{j_{1}} \cdots X_{j_{n}}}\left(\omega_{X_{j_{1}}}, \ldots, \omega_{X_{j_{n}}}\right) \\
\quad+{ }^{c} \mathcal{L}^{X_{j_{1}} \cdots X_{j_{n}}{ }^{*}}\left(-\omega_{X_{j_{1}}}, \ldots,-\omega_{X_{j_{n}}}\right) .
\end{aligned}
$$

Eqs. (30)-(34) determine CC-CI molecular response properties in the elementary basis [Eq. (6)]. The details of the derivation are shown in Ref. 51.

\section{B. CC-CI molecular response properties in diagonal basis}

The eigenvalue equation [Eq. (24)] may alternatively be solved in the diagonal basis [Eq. (17)] where the time evolution of $\left|0_{I}(t, \boldsymbol{\epsilon})\right\rangle$ may be expanded linearly as

$$
\begin{aligned}
\left|0_{I}^{\mathrm{CC}-\mathrm{CI}_{\mathrm{d}}}(t, \boldsymbol{\epsilon})\right\rangle & =e^{W^{\mathrm{CC}}(t, \boldsymbol{\epsilon})}\left|0_{0}^{\mathrm{CC}}\right\rangle \\
& =\left|0_{0}^{\mathrm{CC}}\right\rangle+\sum_{n} w_{n}^{\mathrm{CC}}(t, \boldsymbol{\epsilon})\left|0_{n}^{\mathrm{CC}}\right\rangle,
\end{aligned}
$$

where

$$
W^{\mathrm{CC}}(t, \boldsymbol{\epsilon})=\sum_{n} w_{n}^{\mathrm{CC}}(t, \boldsymbol{\epsilon})\left|0_{n}^{\mathrm{CC}}\right\rangle\left\langle 0_{0}^{\mathrm{CC}}\right|
$$

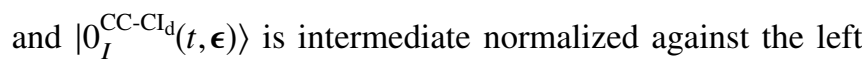
ground state [Eq. (14)],

$$
\left\langle 0_{0}^{\mathrm{CC}} \mid 0_{I}^{\mathrm{CC}-\mathrm{CI}_{\mathrm{d}}}(t, \boldsymbol{\epsilon})\right\rangle=1 .
$$

Substituting Eq. (35) in Eq. (24), multiplying from the left by $e^{-W^{\mathrm{CC}}(t, \boldsymbol{\epsilon})}$ and projecting against $\left\langle\mathcal{B}^{\mathrm{d}}\right|[$ Eq. $(17 \mathrm{~b})]$ in the composite Hilbert space, gives the quasi-energy and the amplitude equations, 


$$
\begin{gathered}
\mathcal{E}(\boldsymbol{\epsilon})=\operatorname{Re}\left\{\left\langle 0_{0}^{\mathrm{CC}}\left|e^{-W^{\mathrm{CC}}(t, \boldsymbol{\epsilon})}\left(H(t, \boldsymbol{\epsilon})-i \frac{\partial}{\partial t}\right) e^{W^{\mathrm{CC}}(t, \boldsymbol{\epsilon})}\right| 0_{0}^{\mathrm{CC}}\right\rangle\right\}_{T}, \\
\left\{\left\langle 0_{n}^{\mathrm{CC}}\left|e^{-W^{\mathrm{CC}}(t, \boldsymbol{\epsilon})}\left(H(t, \boldsymbol{\epsilon})-i \frac{\partial}{\partial t}\right) e^{W^{\mathrm{CC}}(t, \boldsymbol{\epsilon})}\right| 0_{0}^{\mathrm{CC}}\right\rangle\right\}_{T}=0 .
\end{gathered}
$$

As Eq. (25) [or Eq. (27)] and Eq. (35) describe two different representations of the same state, the two sets of equations, Eqs. (29) and (38), have the same solution and give the same quasi-energy.
Molecular response properties may be determined from the quasi-energy Lagrangian referencing the quasienergy and amplitude equations in the diagonal basis [cf. Eq. (38)],

$$
{ }^{c} \mathcal{L}=\left\{\left\langle 0_{0}^{\mathrm{CC}}\left|e^{-W^{\mathrm{CC}}(t, \boldsymbol{\epsilon})}\left(H(t, \boldsymbol{\epsilon})-i \frac{\partial}{\partial t}\right) e^{W^{\mathrm{CC}}(t, \boldsymbol{\epsilon})}\right| 0_{0}^{\mathrm{CC}}\right\rangle\right\}_{T}+\sum_{n}\left\{\bar{w}_{n}^{\mathrm{CC}}(t, \boldsymbol{\epsilon})\left\langle 0_{n}^{\mathrm{CC}}\left|e^{-W^{\mathrm{CC}}(t, \boldsymbol{\epsilon})}\left(H(t, \boldsymbol{\epsilon})-i \frac{\partial}{\partial t}\right) e^{W^{\mathrm{CC}}(t, \boldsymbol{\epsilon})}\right| 0_{0}^{\mathrm{CC}}\right\rangle\right\}_{T},
$$

that satisfies the stationary conditions from which the amplitude and multiplier equations may be determined,

$$
\begin{aligned}
& \frac{\partial^{c} \mathcal{L}}{\partial \bar{w}_{n}^{\mathrm{CC}}\left(\omega_{N}\right)}=0, \\
& \frac{\partial^{c} \mathcal{L}}{\partial w_{n}^{\mathrm{CC}}\left(\omega_{N}\right)}=0,
\end{aligned}
$$

where $w_{n}^{\mathrm{CC}}\left(\omega_{N}\right)$ and $\bar{w}_{n}^{\mathrm{CC}}\left(\omega_{N}\right)$ are amplitudes and multipliers in the frequency domain. CC-CI molecular response properties may be determined in the diagonal basis [Eq. (17)] as described by Eqs. (32)-(34), using the Lagrangian of Eq. (39).

The CC-CI wave functions in Eq. (27) and in Eq. (35) describe the time evolution of the same state in two different bases that span the same space, namely, the elementary basis in Eq. (6) and the diagonal basis in Eq. (17a). We therefore obtain the same molecular response properties whether they are determined from response functions obtained in the elementary or in the diagonal basis, even though the formal expressions for the response functions look very different.

\section{EOM-CC response functions from $\mathrm{Cl}$ response functions}

We will now demonstrate that the EOM-CC response functions obtained by replacing, in the $\mathrm{CI}$ response functions, the CI energies and states with the EOM-CC energies [Eq. (18)] and states [Eqs. (17a) and (17b)] become identical to the CC-CI response functions as expressed in the diagonal basis, provided that the $\mathrm{CI}$ response functions are determined not using the variational condition but using projection (see Ref. 51).

In CI theory, the eigenstates of the Hamiltonian for the unperturbed system, $H_{0}$, are linearly expanded in the basis $|\mathcal{B}\rangle$ [Eq. (2)] and the eigenstates form the basis,

$$
\left|\mathcal{B}_{\mathrm{CI}}^{\mathrm{d}}\right\rangle=\left\{\left|0_{0}^{\mathrm{CI}}\right\rangle,\left|0_{n}^{\mathrm{CI}}\right\rangle, \quad n>0\right\},
$$

that satisfies

$$
\begin{gathered}
\left\langle 0_{p}^{\mathrm{CI}}\left|H_{0}\right| 0_{q}^{\mathrm{CI}}\right\rangle={ }^{\mathrm{CI}} E_{q} \delta_{p q}, \quad p, q=0,1,2, \ldots, \\
\left\langle 0_{p}^{\mathrm{CI}} \mid 0_{q}^{\mathrm{CI}}\right\rangle=\delta_{p q}, \quad p, q=0,1,2, \ldots
\end{gathered}
$$

The time evolution of $\left|0_{I}(t, \boldsymbol{\epsilon})\right\rangle$ may be linearly expanded in the diagonal CI basis, $\left|\mathcal{B}_{\mathrm{CI}}^{\text {d }}\right\rangle$ [Eq. (41)],

$\left|0_{I}^{\mathrm{CI}}(t, \boldsymbol{\epsilon})\right\rangle=e^{W^{\mathrm{CI}}(t, \boldsymbol{\epsilon})}\left|0_{0}^{\mathrm{CI}}\right\rangle=\left|0_{0}^{\mathrm{CI}}\right\rangle+\sum_{n} w_{n}^{\mathrm{CI}}(t, \boldsymbol{\epsilon})\left|0_{n}^{\mathrm{CI}}\right\rangle$,

where

$$
W^{\mathrm{CI}}(t, \boldsymbol{\epsilon})=\sum_{n} w_{n}^{\mathrm{CI}}(t, \boldsymbol{\epsilon})\left|0_{n}^{\mathrm{CI}}\right\rangle\left\langle 0_{0}^{\mathrm{CI}}\right| .
$$

$\left|0_{I}^{C \mathrm{I}_{\mathrm{d}}}(t, \boldsymbol{\epsilon})\right\rangle$ is intermediate normalized against the ground state,

$$
\left\langle 0_{0}^{\mathrm{CI}} \mid 0_{I}^{\mathrm{CI}_{\mathrm{d}}}(t, \boldsymbol{\epsilon})\right\rangle=1 .
$$

Note the similarity between the time evolution of the CI state described by Eqs. (43)-(45) and the time evolution of the CC-CI state in Eqs. (35)-(37). Substituting Eq. (43) in Eq. (24), multiplying from the left by $e^{-W^{\mathrm{Cl}}(t, \epsilon)}$ and projecting against the basis

$$
\left\langle\mathcal{B}_{\mathrm{CI}}^{\mathrm{d}}\right|=\left(\left|\mathcal{B}_{\mathrm{CI}}^{\mathrm{d}}\right\rangle\right)^{\dagger}=\left\{\left\langle 0_{0}^{\mathrm{CI}}\right|,\left\langle 0_{n}^{\mathrm{CI}}\right|, \quad n>0\right\}
$$

in the composite Hilbert space, gives equations similar to Eq. (38) where the label CC is replaced by the label CI. In CI theory, molecular response functions may therefore be derived from the Lagrangian in Eq. (39) where the label CC is replaced by the label CI.

In deriving the CI response functions, simplifications occur in the Lagrangian for the term containing the unperturbed Hamiltonian, $H_{0}$, since Eq. (42a) is satisfied. ${ }^{51}$ For the CC-CI Lagrangian [Eq. (39)], similar simplifications may be introduced since Eq. (18a) is satisfied. In CI theory, we further have that

$$
\left\langle 0_{n}^{\mathrm{CI}}\left|H_{0}\right| 0_{0}^{\mathrm{CI}}\right\rangle^{\dagger}=\left\langle 0_{0}^{\mathrm{CI}}\left|H_{0}\right| 0_{n}^{\mathrm{CI}}\right\rangle, \quad n>0,
$$

which has no counterpart in $\mathrm{CC}$ theory,

$$
\left\langle 0_{n}^{\mathrm{CC}}\left|H_{0}\right| 0_{0}^{\mathrm{CC}}\right\rangle^{\dagger} \neq\left\langle 0_{0}^{\mathrm{CC}}\left|H_{0}\right| 0_{n}^{\mathrm{CC}}\right\rangle, \quad n>0 .
$$


However, Eq. (47) cannot be used in CI theory to obtain simplifications when the CI response functions are derived. The CC-CI Lagrangian in the diagonal basis of Eq. (17) and the CI Lagrangian in the diagonal basis of Eq. (41) have therefore the same structural form. Consequently, the CC-CI Lagrangian in the diagonal basis may be obtained from the CI Lagrangian replacing the CI eigenvalues and eigenstates of Eqs. (41) and (46) by the EOM-CC eigenvalues and eigenstates of Eqs. (17a) and (17b). A presumption is of course that the CI response functions are determined via projection, as it is described above, and thus contain the $1 / 2 C^{ \pm \omega}$ operator of Eq. (34).

The EOM-CC response functions introduced by Stanton an Bartlett $^{2}$ will thus become identical to the CC-CI response functions in the diagonal basis. Since CC-CI molecular response properties are independent of the basis in which they are expressed if the bases span the same space, the EOM$\mathrm{CC}$ response functions will thus give molecular response properties that are identical to the CC-CI molecular response properties in the elementary basis. This is also the case for molecular response properties that are determined from residues of the response functions.

In Ref. 51, we showed explicitly that the EOM-CC linear response function and the CC-CI linear response function in the elementary basis give identical molecular response properties, which required a substantial rewrite of the involved equations. However, we did not recognize that the equivalence between the EOM-CC and CC-CI was also valid for quadratic and higher-order response functions, as has been shown in this section.

The EOM-CC molecular response properties may thus be determined from the CC-CI response functions and from the residues of these response functions. For static perturbations, the $\mathrm{CC}$-CI molecular response properties are also identical to the molecular response properties that are obtained from the finite-field EOM-CC energy calculations, as discussed in Sec. III. The CC-CI model thus constitutes a first principle and simple time-dependent framework for deriving EOM-CC molecular response properties.

TABLE I. Polarizability $(\alpha)$, first hyperpolarizability $(\beta)$, and transition strengths $\left(\left|M_{0 m}\right|^{2}\right)$ for the lowest allowed dipole transition of excitation energy $\omega_{m}=\left(E_{m}-E_{0}\right)$, calculated in CC-CISD, EOM-CCSD, and standard CCSD models using a cc-pVDZ basis. ${ }^{70,71}$ As discussed in the text, analytical CC-CISD and EOM-CCSD results have been obtained using two independent implementations. However, as they yield the same numbers, analytical CC-CISD and EOM-CCSD results are presented together in the fourth column. For frequency-dependent properties, the frequency $\omega=0.07200275$ a.u., corresponding to the wavelength $\lambda=632.8 \mathrm{~nm}$, is used, and the second-harmonic generation process is calculated for $\beta(\omega)$. EOM-CCSD finite field results are obtained by numerical differentiation of $E_{0}(\epsilon)$ of Eq. (20) (see text for details). Internuclear distances are given in the text. All results in a.u.

\begin{tabular}{|c|c|c|c|c|}
\hline Molecule & Property & $\begin{array}{c}\text { CCSD } \\
\text { analytical }\end{array}$ & $\begin{array}{c}\text { CC-CISD/EOM-CCSD } \\
\text { analytical }\end{array}$ & $\begin{array}{l}\text { EOM-CCSD } \\
\text { finite field }\end{array}$ \\
\hline \multirow[t]{6}{*}{$\mathrm{HeH}^{+}$} & $\alpha_{z z}(0)$ & 1.5165613 & 1.5165613 & \multirow[t]{2}{*}{$1.5165613^{b}$} \\
\hline & $\alpha_{z z}(\omega)$ & 1.5243799 & 1.5243799 & \\
\hline & $\beta_{z z z}(0)$ & 2.7354205 & 2.7354205 & \multirow[t]{4}{*}{$2.7354203^{\circ}$} \\
\hline & $\beta_{z z z}(\omega)$ & 2.8268468 & 2.8268468 & \\
\hline & $\left|M_{0 m}\right|^{2}$ & 0.6035931 & 0.6035931 & \\
\hline & $\omega_{m}$ & 0.9543351 & 0.9543351 & \\
\hline \multirow[t]{4}{*}{$\mathrm{H}_{2}$} & $\alpha_{z z}(0)$ & 6.3283056 & 6.3283056 & \multirow[t]{4}{*}{$6.3283056^{\circ}$} \\
\hline & $\alpha_{z z}(\omega)$ & 6.4509846 & 6.4509846 & \\
\hline & $\left|M_{0 m}\right|^{2}$ & 1.5325340 & 1.5325340 & \\
\hline & $\omega_{m}$ & 0.5111570 & 0.5111570 & \\
\hline \multirow[t]{6}{*}{$\mathrm{HF}$} & $\alpha_{z z}(0)$ & 4.2052174 & 4.2303230 & \multirow[t]{2}{*}{$4.2303230^{\circ}$} \\
\hline & $\alpha_{z z}(\omega)$ & 4.2453024 & 4.2706176 & \\
\hline & $\beta_{z z z}(0)$ & 13.269879 & 13.285769 & \multirow[t]{4}{*}{$13.28580^{\mathrm{f}}$} \\
\hline & $\beta_{z z z}(\omega)$ & 14.455227 & 14.487462 & \\
\hline & $\left|M_{0 m}\right|^{2}$ & 0.6333985 & 0.6365062 & \\
\hline & $\omega_{m}$ & 0.5736772 & 0.5736772 & \\
\hline \multirow[t]{4}{*}{$\mathrm{N}_{2}$} & $\alpha_{z z}(0)$ & 12.810904 & 13.058818 & \multirow[t]{4}{*}{$13.058816^{\mathrm{g}}$} \\
\hline & $\alpha_{z z}(\omega)$ & 12.935645 & 13.186343 & \\
\hline & $\left|M_{0 m}\right|^{2}$ & 1.8611090 & 1.9158357 & \\
\hline & $\omega_{m}$ & 0.6245830 & 0.6245830 & \\
\hline
\end{tabular}

a Numerical derivatives were calculated using Newton's forward interpolation formulas ${ }^{75}$ containing $n$ terms with $n=1,2, \ldots, 20$.

A scan over 20 different values of the differencing interval $(h)$ has been performed for each formula. In this way optimal values of $h$ and $n$, in the sense of yielding the smallest uncertainty $\Delta$, have been found for each molecule and property.

${ }^{\mathrm{b}} h=0.01$ a.u., $n=7, \Delta \approx 2 \times 10^{-9}$ a.u.

${ }^{\mathrm{c}} h=0.01$ a.u., $h=7, \Delta \approx 2 \times 10^{-7}$ a.u.

${ }^{\mathrm{d}} h=0.01$ a.u., $h=9, \Delta \approx 6 \times 10^{-9}$ a.u.

${ }^{\mathrm{e}} h=0.01$ a.u., $n=8, \Delta \approx 8 \times 10^{-8}$ a.u.

${ }^{\mathrm{f}} h=0.01$ a.u., $n=7, \Delta \approx 3 \times 10^{-5}$ a.u.

${ }^{\mathrm{g}} h=0.001$ a.u., $n=3, \Delta \approx 2 \times 10^{-6}$ a.u. 


\section{ILLUSTRATIVE CALCULATIONS}

In this section, we present numerical illustration of the theoretical findings discussed in Secs. II-IV. For comparison, we also report results for molecular response properties calculated using standard CC response theory. ${ }^{14,49,51}$

We will report calculations of frequency-dependent polarizabilities $(\alpha)$, first hyperpolarizabilities $(\beta)$, and transition strengths $\left(\left|M_{0 m}\right|^{2}\right)$ for the lowest allowed dipole transition of excitation energy $\omega_{m}=E_{m}-E_{0}$, for $\mathrm{HeH}^{+}$, $\mathrm{H}_{2}, \mathrm{HF}$, and $\mathrm{N}_{2}$ molecules at internuclear distances of 1.5 a.u., 1.401214813 a.u., ${ }^{69} 1.732650075$ a.u., ${ }^{69}$ and 2.074408909 a.u. ${ }^{69}$ respectively, using a cc-pVDZ basis. ${ }^{70,71}$ The calculations have been performed using a development version of the Dalton programme suite. ${ }^{72,73}$ The expressions that have been implemented to compute the CC-CI and EOM$\mathrm{CC}$ polarizabilities and first hyperpolarizabilities are given in Ref. 51. The expressions that have been implemented for transition moments are given in Ref. 74. The finite field EOM$\mathrm{CC}$ eigenvalue equation calculations have been performed as described in Sec. III and numerical derivatives have been obtained applying Newton's forward interpolation formulas ${ }^{75}$ to the EOM-CC field-dependent energy of Eq. (20). Estimates will also be given of the uncertainties of the numerical derivatives.

The results of the calculations are given in Table I. We first note that the numerical values of the polarizabilities, hyperpolarizabilities, and transition strengths calculated using the implementation of either the CC-CI or the EOM-CC analytic expressions are identical and are therefore presented together in the fourth column of Table I. The expressions that have been implemented for the CC-CI polarizabilities and first hyperpolarizabilities are Eqs. (340) and (343) of Ref. 51, respectively. The EOM-CC polarizability and first hyperpolarizability expressions are Eqs. (407) and (408) of Ref. 51. For transition strengths, the CC-CI and EOM-CC expressions in Ref. 74 have been implemented. Note again that the CC-CI and EOM-CC expressions for a molecular property look very different. As we have shown in Sec. IV C, however, these different expressions lead in CC-CI and EOM$\mathrm{CC}$ theory to identical molecular response properties, as also confirmed by the calculations reported in Table I.

Finite field EOM-CC results for static molecular response properties are also given in Table I together with estimated uncertainties of the numerical derivatives. The uncertainty of the numerical differentiation of course increases for the higher derivatives. However, the finite field results in Table I clearly demonstrate that the numerical differentiation of the EOM-CC field-dependent energy of Eq. (20) corresponds exactly to the analytic $\mathrm{CC}-\mathrm{CI} / \mathrm{EOM}-\mathrm{CC}$ static molecular response properties.

In Table I, we also give results for standard CC response calculations, i.e., calculations where an exponential parametrization is used to describe both the unperturbed system and its time evolution. For two-electron systems, CC theory containing all single and double excitations gives FCI results, independent of whether a linear or an exponential parametrization is used to describe the time evolution of the unperturbed state. The CCSD and CC-CISD/EOM-CCSD results in Table I are therefore identical for $\mathrm{HeH}^{+}$and $\mathrm{H}_{2}$. For
$\mathrm{HF}$ and $\mathrm{N}_{2}$, the CC-CISD/EOM-CCSD results differ from the CCSD results but the differences are relatively small (up to a few percent). This reflects that small changes are introduced in a molecular system by applying the time-periodic perturbation and that these small changes for small molecular systems can be described quite well by a truncated linear expansion. Note also that the excitation energies are the same for the CCSD and CC-CISD/EOM-CCSD models, not only for two-electron systems but also for systems of an arbitrary size.

\section{SUMMARY AND CONCLUSIONS}

EOM-CC molecular response properties are commonly obtained by taking the CI response functions (and residues of the CI response functions) and replacing the energies and eigenstates of the $\mathrm{CI}$ eigenvalue equation with the energies and eigenstates of the EOM-CC eigenvalue equation. ${ }^{2} \mathrm{We}$ have here demonstrated that EOM-CC molecular response properties are identical to the ones determined using a CC-CI time-dependent framework, also when truncations are introduced in the excitation manifold. However, the equivalence holds only if the $\mathrm{CI} \rightarrow$ EOM-CC replacement of energies and eigenstates is performed in the CI response functions (and residues of the CI response functions) obtained using projection, and not in those obtained using the variational principle. The equivalence between EOM-CC and CC-CI response properties holds for all orders of the response functions and not, as incorrectly stated in Ref. 51, only for molecular response properties that can be described in terms of linear response functions.

The CC-CI approach offers a first-principle, timedependent perspective to derive the EOM-CC molecular response property expressions and to analyze and understand the basic assumptions on which these expressions are founded. The equivalence between EOM-CC and CC-CI molecular response properties also facilitates the determination of EOM$\mathrm{CC}$ static molecular response properties from finite-field EOM-CC energy calculations.

In CC-CI theory, the time-dependent Schrödinger equation is solved using an exponential parametrization to describe the unperturbed state and a linear parametrization to describe the time evolution of this state. As the time-dependent Schrödinger equation is solved in CC-CI theory, EOM-CC molecular response properties become identical to FCI molecular response properties when no truncation is carried out in the excitation manifold in the CC-CI calculations.

In standard CC response theory, both the unperturbed system and its time evolution are described in terms of an exponential parametrization and size extensive molecular response properties are therefore obtained. In CC-CI theory, the time evolution of the unperturbed state is described in terms of a linear parametrization and the EOM-CC molecular response properties therefore are not size extensive when truncations are carried out in the excitation manifold that enters the EOM-CC eigenvalue equation. For small molecular systems, there is little difference between describing the time evolution of the unperturbed system in terms of an exponential or a linear truncated expansion since the energy contributions from the added time-periodic perturbation are small compared 
to the ground-state total energy. The size-extensivity problems therefore are not severe for small molecular systems and the deviations between molecular response properties that are calculated using CC-CI/EOM-CC and conventional CC theory are small for small molecular systems. For large molecular systems, the size-extensivity problems may become severe and lead to non-physical results, e.g., negative transition strengths, as shown in Refs. 15 and 76.

\section{ACKNOWLEDGMENTS}

S.C. acknowledges financial support from the AIAS-COFUND program (Grant Agreement No. 609033). F.P. and P.J. acknowledge support from The European Research Council under the European Union's (EU) Seventh Framework Programme (FP/2007-2013)/ERC Grant Agreement No. 291371 and P.J. and J.O. acknowledge support from The Danish Council for Independent Research — Natural Sciences. J.O. also acknowledges support from the Danish Council for Independent Research, No. DFF-4181-00537. Calculations have been carried out using resources provided by Wroclaw Centre for Networking and Supercomputing (http://www.wcss.pl/en/), Grant No. 277.

${ }^{1}$ D. C. Comeau and R. J. Bartlett, Chem. Phys. Lett. 207, 414 (1993).

${ }^{2}$ J. F. Stanton and R. J. Bartlett, J. Chem. Phys. 98, 7029 (1993).

${ }^{3}$ I. Shavitt and R. J. Bartlett, Many-Body Methods in Chemistry and Physics: MBPT and Coupled-Cluster Theory (Cambridge University Press, 2009).

${ }^{4}$ M. Nooijen and R. J. Bartlett, J. Chem. Phys. 106, 6441 (1997).

${ }^{5}$ P. Piecuch and R. J. Bartlett, Adv. Quantum Chem. 34, 295 (1999).

${ }^{6}$ A. I. Krylov, Chem. Phys. Lett. 338, 375 (2001).

${ }^{7}$ M. Kállay and J. Gauss, J. Chem. Phys. 121, 9257 (2004).

${ }^{8}$ S. V. Levchenko and A. I. Krylov, J. Chem. Phys. 120, 175 (2004).

${ }^{9}$ S. Nangia, D. G. Truhlar, M. J. McGuire, and P. Piecuch, J. Phys. Chem. A 109, 11643 (2005).

${ }^{10}$ Y. Ohtsuka, P. Piecuch, J. R. Gour, M. Ehara, and H. Nakatsuji, J. Chem. Phys. 126, 164111 (2007).

${ }^{11}$ L. Kong, K. R. Shamasundar, O. Demel, and M. Nooijen, J. Chem. Phys. 130, 114101 (2009).

${ }^{12}$ P. Piecuch, J. R. Gour, and M. Włoch, Int. J. Quantum Chem. 109, 3268 (2009).

${ }^{13}$ J. Sous, P. Goel, and M. Nooijen, Mol. Phys. 112, 616 (2013).

${ }^{14}$ H. Koch and P. Jørgensen, J. Chem. Phys. 93, 3333 (1990).

${ }^{15}$ H. Koch, R. Kobayashi, A. Sánchez de Merás, and P. Jørgensen, J. Chem. Phys. 100, 4393 (1994).

${ }^{16}$ G. D. Purvis and R. J. Bartlett, J. Chem. Phys. 76, 1910 (1982).

${ }^{17}$ J. Noga and R. J. Bartlett, J. Chem. Phys. 86, 7041 (1987); Erratum, 89, 3401 (1988).

${ }^{18}$ G. E. Scuseria and H. F. Schaefer III, Chem. Phys. Lett. 152, 382 (1988).

${ }^{19}$ N. Oliphant and L. Adamowicz, J. Chem. Phys. 95, 6645 (1991).

${ }^{20}$ S. A. Kucharski and R. J. Bartlett, J. Chem. Phys. 97, 4282 (1992).

${ }^{21}$ J. Olsen, J. Chem. Phys. 113, 7140 (2000).

${ }^{22}$ M. Kállay and P. R. Surján, J. Chem. Phys. 113, 1359 (2000).

${ }^{23}$ H. Koch, H. J. A. Jensen, P. Jørgensen, and T. Helgaker, J. Chem. Phys. 93, 3345 (1990).

${ }^{24}$ K. Hald, P. Jørgensen, J. Olsen, and M. Jaszuński, J. Chem. Phys. 115, 671 (2001).

${ }^{25}$ S. Hirata, J. Chem. Phys. 121, 51 (2004).

${ }^{26}$ J. F. Stanton and J. Gauss, J. Chem. Phys. 103, 1064 (1995).

${ }^{27}$ S. R. Gwaltney, M. Nooijen, and R. J. Bartlett, Chem. Phys. Lett. 248, 189 (1996).

${ }^{28}$ M. Head-Gordon, M. Oumi, and D. Maurice, Mol. Phys. 96, 593 (1999).

${ }^{29}$ T. D. Crawford and R. A. King, Chem. Phys. Lett. 366, 611 (2002).

${ }^{30}$ S. Hirata, P.-D. Fan, T. Shiozaki, and Y. Shigeta, in Radiation Induced Molecular Phenomena in Nucleic Acids, Challenges and Advances in Computational Chemistry and Physics, edited by M. K. Shukla and J. Leszczynski (Springer, Netherlands, 2008), Vol. 5, pp. 15-64.

${ }^{31}$ G. Fradelos, J. J. Lutz, T. A. Wesołowski, P. Piecuch, and M. Włoch, Adv. Theory Quantum Syst. Chem. Phys. 22, 219 (2012).
${ }^{32}$ J. J. Goings, M. Caricato, M. J. Frisch, and X. Li, J. Chem. Phys. 141, 164116 (2014).

${ }^{33}$ R. J. Bartlett, WIREs Comput. Mol. Sci. 2, 126 (2012).

${ }^{34}$ A. I. Krylov, Annu. Rev. Phys. Chem. 59, 433 (2008).

${ }^{35}$ S. Pal, M. Rittby, R. J. Bartlett, D. Sinha, and D. Mukherjee, Chem. Phys. Lett. 137, 273 (1987).

${ }^{36}$ J. F. Stanton and J. Gauss, J. Chem. Phys. 101, 8938 (1994).

${ }^{37}$ M. Nooijen and R. J. Bartlett, J. Chem. Phys. 102, 3629 (1995).

${ }^{38}$ K. W. Sattelmeyer, H. F. Schaefer III, and J. F. Stanton, Chem. Phys. Lett. 378, 42 (2003).

${ }^{39}$ J. R. Gour, P. Piecuch, and M. Włoch, J. Chem. Phys. 123, 134113 (2005).

${ }^{40}$ J. R. Gour, P. Piecuch, and M. Włoch, Int. J. Quantum Chem. 106, 2854 (2006).

${ }^{41}$ M. Kamiya and S. Hirata, J. Chem. Phys. 126, 134112 (2007).

${ }^{42}$ N. Bloembergen, Nonlinear Optics, 4th ed. (World Scientific Publishing Company, Inc., Singapore, 1996).

${ }^{43}$ Non-Linear Raman Spectroscopy and Its Chemical Aplications, edited by W. Kiefer and D. A. Long (Springer, Dordrecht, Netherlands, 1982).

${ }^{44}$ D. P. Craig and T. Thirunamachandran, Molecular Quantum Electrodynamics (Academic Press, New York, 1984).

${ }^{45}$ Nonlinear Optical Properties of Organic Molecules and Crystals, edited by D. S. Chemla and J. Zyss (Academic Press, New York, 1987).

${ }^{46}$ T. Kobayashi, in Nonlinear Optics of Organics and Semiconductors: Proceedings of the International Symposium, Tokyo, Japan, 25-26 July 1988 (Springer-Verlag, Berlin, 1989).

${ }^{47}$ D. N. Zubarev, Nonlinear Statistical Thermodynamics (Consultant Bureau, Plenum, New York, 1974).

${ }^{48}$ J. Olsen and P. Jørgensen, J. Chem. Phys. 82, 3235 (1985).

${ }^{49}$ O. Christiansen, P. Jørgensen, and C. Hättig, Int. J. Quantum Chem. 68, 1 (1998).

${ }^{50}$ T. Helgaker, S. Coriani, P. Jørgensen, K. Kristensen, J. Olsen, and K. Ruud, Chem. Rev. 112, 543 (2012).

${ }^{51}$ F. Pawłowski, J. Olsen, and P. Jørgensen, J. Chem. Phys. 142, 114109 (2015).

${ }^{52}$ M. Göppert-Mayer, Ann. Phys. 401, 273 (1931).

${ }^{53}$ J. F. Stanton and R. J. Bartlett, J. Chem. Phys. 99, 5178 (1993).

${ }^{54}$ R. Kobayashi, H. Koch, and P. Jørgensen, Chem. Phys. Lett. 219, 30 (1994).

${ }^{55}$ P. B. Rozyczko, S. A. Perera, M. Nooijen, and R. J. Bartlett, J. Chem. Phys. 107, 6736 (1997).

${ }^{56}$ P. Rozyczko and R. J. Bartlett, J. Chem. Phys. 107, 10823 (1997).

${ }^{57}$ P. B. Rozyczko and R. J. Bartlett, J. Chem. Phys. 108, 7988 (1998).

${ }^{58}$ K. D. Nanda and A. I. Krylov, J. Chem. Phys. 142, 064118 (2015).

${ }^{59}$ S. A. Perera, M. Nooijen, and R. J. Bartlett, J. Chem. Phys. 104, 3290 (1996).

${ }^{60}$ A. Ye, Z. Shuai, O. Kwon, J. L. Bredas, and D. Beljonne, J. Chem. Phys. 121, 5567 (2004).

${ }^{61}$ Z. Shuai and J. L. Bredas, Phys. Rev. B 62, 15452 (2000).

${ }^{62}$ S. A. Perera and R. J. Bartlett, Mol. Phys. 104, 2403 (2006).

${ }^{63}$ S. A. Perera and R. J. Bartlett, Chem. Phys. Lett. 314, 381 (1999).

${ }^{64}$ T. F. Hughes and R. J. Bartlett, J. Chem. Phys. 129, 054105 (2008).

${ }^{65}$ T. D. Crawford and H. Sekino, in Advances in the Theory of Atomic and Molecular Systems: Conceptual and Computational Advances in Quantum Chemistry, edited by P. Piecuch, J. Maruani, G. Delgado Barrio, and S. Wilson (Springer, Dordrecht, 2009), Vol. 19, pp. 225-239.

${ }^{66}$ H. R. McAlexander and T. D. Crawford, J. Chem. Phys. 142, 154101 (2015).

${ }^{67}$ T. Helgaker, P. Jørgensen, and J. Olsen, Molecular Electronic-Structure Theory (Wiley, Chichester, 2000).

${ }^{68}$ H. Sambe, Phys. Rev. A 7, 2203 (1973).

${ }^{69}$ F. Pawłowski, P. Jørgensen, J. Olsen, F. Hegelund, T. Helgaker, J. Gauss, K. L. Bak, and J. F. Stanton, J. Chem. Phys. 116, 6482 (2002).

${ }^{70}$ T. H. Dunning, Jr., J. Chem. Phys. 90, 1007 (1989).

${ }^{71}$ D. E. Woon and T. H. Dunning, Jr., J. Chem. Phys. 100, 2975 (1994).

${ }^{72}$ K. Aidas, C. Angeli, K. L. Bak, V. Bakken, R. Bast, L. Boman, O. Christiansen, R. Cimiraglia, S. Coriani, P. Dahle, E. K. Dalskov, U. Ekström, T. Enevoldsen, J. J. Eriksen, P. Ettenhuber, B. Fernández, L. Ferrighi, H. Fliegl, L. Frediani, K. Hald, A. Halkier, C. Hättig, H. Heiberg, T. Helgaker, A. C. Hennum, H. Hettema, E. Hjertenæs, S. Høst, I.-M. Høyvik, M. F. Iozzi, B. Jansík, H. J. A. Jensen, D. Jonsson, P. Jørgensen, J. Kauczor, S. Kirpekar, T. Kjærgaard, W. Klopper, S. Knecht, R. Kobayashi, H. Koch, J. Kongsted, A. Krapp, K. Kristensen, A. Ligabue, O. B. Lutnæs, J. I. Melo, K. V. Mikkelsen, R. H. Myhre, C. Neiss, C. B. Nielsen, P. Norman, J. Olsen, J. M. H. Olsen, A. Osted, M. J. Packer, F. Pawłowski, T. B. Pedersen, P. F. Provasi, S. Reine, Z. Rinkevicius, T. A. Ruden, K. Ruud, V. V. Rybkin, P. Sałek, C. C. M. Samson, A. Sánchez de Merás, T. Saue, S. P. A. Sauer, B. Schimmelpfennig, K. Sneskov, A. H. Steindal, K. O. Sylvester-Hvid, P. R. Taylor, A. M. Teale, E. I. Tellgren, D. P. Tew, A. J. Thorvaldsen, L. Thøgersen, O. Vahtras, M. A. Watson, D. J. D. Wilson, M. Ziółkowski, and H. Ågren, WIREs Comput. Mol. Sci. 4, 269 (2014). 
${ }^{73}$ Dalton, a Molecular Electronic Structure Program, Release DALTON2015.1, 2015, see http://daltonprogram.org/.

${ }^{74} \mathrm{CC}$-CI transition strengths are obtained by taking a residue of the linear response function and, in the notation of Ref. 51, are given by

$$
\begin{aligned}
\left|M_{0 m}\right|^{2} & =\lim _{\omega_{X_{j_{2}} \rightarrow \omega_{m}}}\left(\omega_{X_{j_{2}}}-\omega_{m}\right)\left\langle\left\langle X_{j_{1}} ; X_{j_{2}}\right\rangle\right\rangle_{\omega_{X_{j_{2}}}} \\
& =\frac{1}{2}\left[S_{0 m}^{X_{j_{1}} X_{j_{2}}}+\left(S_{0 m}^{X_{j_{2}} X_{j_{1}}}\right)^{*}\right],
\end{aligned}
$$

where $\omega_{m}=\left(E_{m}-E_{0}\right)$ is an excitation energy corresponding to an excited state $\mathbf{C}_{m}^{\perp}$ and

$$
S_{0 m}^{X_{j_{1}} X_{j_{2}}}=T_{0 m}^{X_{j_{1}}} T_{m 0}^{X_{j_{2}}} .
$$

The CC-CI transition moments read

$$
\begin{gathered}
T_{0 m}^{X_{j_{1}}}=\sum_{v_{n}}\left[\left(-\sum_{\mu_{k}} \bar{t}_{\mu_{k}}^{(0)} s_{\mu_{k}}^{X_{j_{1}}}\left(-\omega_{m}\right)\right)\left\langle\mathrm{HF}\left|H_{0}^{T_{0}}\right| v_{n}\right\rangle\right. \\
+\left(-\sum_{\mu_{k}}\left\langle\mathrm{HF}\left|H_{0}^{T_{0}}\right| \mu_{k}\right\rangle s_{\mu_{k}}^{X_{j_{1}}}\left(-\omega_{m}\right)\right) \bar{t}_{v_{n}}^{(0)}+\left\langle\mathrm{HF}\left|X_{j_{1}}^{T_{0}}\right| v_{n}\right\rangle \\
\left.+\sum_{\mu_{k}} \bar{t}_{\mu_{k}}^{(0)}\left\langle\mu_{k}\left|\left[X_{j_{1}}^{T_{0}},\left|v_{n}\right\rangle\langle\mathrm{HF}|\right]\right| \mathrm{HF}\right\rangle\right] C_{v_{n} m}^{\perp}, \\
T_{m 0}^{X_{j_{2}}}=\sum_{v_{n}} \bar{C}_{m v_{n}}^{\perp}\left\langle v_{n}\left|X_{j_{2}}^{T_{0}}\right| \mathrm{HF}\right\rangle .
\end{gathered}
$$

EOM-CC transition strengths are obtained by taking the CI transition strength expressions (in the diagonal representation) and replacing the eigenstates of the CI eigenvalue equation with the eigenstates of the EOM-CC eigenvalue equation,

$$
\left|M_{0 m}\right|^{2}=\left\langle 0_{0}^{\mathrm{CC}}\left|X_{j_{1}}\right| 0_{m}^{\mathrm{CC}}\right\rangle\left\langle 0_{m}^{\mathrm{CC}}\left|X_{j_{2}}\right| 0_{0}^{\mathrm{CC}}\right\rangle,
$$

and the EOM-CC transition moments consequently read

$$
\begin{aligned}
T_{0 m}^{X_{j_{1}}}= & \left\langle 0_{0}^{\mathrm{CC}}\left|X_{j_{1}}\right| 0_{m}^{\mathrm{CC}}\right\rangle \\
= & \sum_{v_{n}}\left[-\left(\sum_{\mu_{k}} \bar{t}_{\mu_{k}}^{(0)}\left\langle\mu_{k}\left|X_{j_{1}}^{T_{0}}\right| \mathrm{HF}\right\rangle\right) \bar{t}_{v_{n}}^{(0)}\right. \\
& \left.+\left\langle\mathrm{HF}\left|X_{j_{1}}^{T_{0}}\right| v_{n}\right\rangle+\sum_{\mu_{k}} \bar{t}_{\mu_{k}}^{(0)}\left\langle\mu_{k}\left|\left[X_{j_{1}}^{T_{0}},\left|v_{n}\right\rangle\langle\mathrm{HF}|\right]\right| \mathrm{HF}\right\rangle\right] C_{v_{n} m}^{\perp}, \\
T_{m 0}^{X_{j_{2}}}= & \left\langle 0_{m}^{\mathrm{CC}}\left|X_{j_{2}}\right| 0_{0}^{\mathrm{CC}}\right\rangle=\sum_{v_{n}} \bar{C}_{m v_{n}}^{\perp}\left\langle v_{n}\left|X_{j_{2}}^{T_{0}}\right| \mathrm{HF}\right\rangle .
\end{aligned}
$$

The EOM-CC and CC-CI expressions for right transition moments $T_{m 0}^{X_{j_{2}}}$ are identical, whereas those for the left moments $T_{0 m}^{X_{j_{1}}}$ look different. However, by modifying the left CC-CI transition moment expression to comply with the $2 n+1$ rule, an expression identical to the EOM-CC expression is obtained.

${ }^{75}$ G. S. Rao, Numerical Analysis (New Age International, 2006).

${ }^{76}$ M. Caricato, G. W. Trucks, and M. J. Frisch, J. Chem. Phys. 131, 174104 (2009) 